\title{
Ventricular Tachycardia Ablation in Patients with HeartMate II Left Ventricular Assist Devices: Rhythm Still Matters in the Bionic Age
}

\author{
${ }^{1}$ RASHMI U. HOTTIGOUDAR, MD, ${ }^{1}$ A. GREGORY DEAM, MD, ${ }^{2}$ MARK S. SLAUGHTER, MD, \\ ${ }^{1}$ BRAD S. SUTTON, MD, MBA, ${ }^{1}$ KELLY MCCANTS, MD, ${ }^{1}$ EMMA BIRKS, MD, PhD and \\ ${ }^{1}$ RAKESH GOPINATHANNAIR, MD, MA
}

Divisions of ${ }^{1}$ Cardiovascular Medicine and ${ }^{2}$ Cardiothoracic Surgery, University of Louisville, Louisville, $K Y$

\begin{abstract}
Patients with long-term continuous flow left ventricular assist devices (LVADs) are at increased risk of developing ventricular arrhythmias, which can result in defibrillator shocks and right ventricular dysfunction. We report a series of three patients with continuous flow HeartMate II LVADs who underwent catheter ablation for incessant, medically refractory VTs after a median of 26 months following LVAD implant. Our experience shows that endocardial catheter ablation can be safely and effectively performed provided careful attention is paid towards catheter manipulation and LVAD function. Although the LVAD provides hemodynamic stability allowing detailed mapping during $V T$, extensive arrhythmogenic endocardial substrate is usually encountered with multiple inducible VT morphologies. More than one procedure may be required and moderate long-term VT control can be achieved with a combination of catheter ablation and antiarrhythmic therapy.
\end{abstract}

KEYWORDS. catheter ablation, left ventricular assist device, ventricular tachycardia.
ISSN 2156-3977 (print) ISSN 2156-3993 (online)

(C) 2011 Innovations in Cardiac Rhythm Management

\section{Introduction}

Heart transplantation remains the most successful longterm therapeutic option for patients with end-stage heart failure. ${ }^{1}$ Despite its efficacy, the demand for donor hearts far exceeds the availability. This limited availability, coupled with the increasing comorbidities of heart failure patients rendering them unsuitable for transplantation, has resulted in a rapid increase in left ventricular assist device (LVAD) therapy, both as a bridge-to-transplant as well as bridge-to-recovery, and, not infrequently, as destination therapy. ${ }^{2}$

LVADs have been increasingly used to improve hemodynamic status in patients with refractory ventricular arrhythmias (VTs), especially in those patients

The authors report no conflicts of interest for the published content. Manuscript received September 29, 2011, final version accepted October 14, 2011.

Address correspondence to: Rakesh Gopinathannair, MD, MA, Director of Cardiac Electrophysiology, University of Louisville Hospital, Assistant Professor of Medicine, Division of Cardiology, University of Louisville, ACB/A3L42, 550 So. Jackson St. Louisville, KY. E-mail: r0gopi01@louisville.edu undergoing catheter ablation of unmappable VTs. ${ }^{3,4}$ However, LVAD recipients are at an increased risk of developing new-onset monomorphic VT, irrespective of whether or not they had VT prior to LVAD implantation. ${ }^{5}$ Current generation HeartMate II (Thoratec Corporation, Pleasanton, CA) devices require adequate LV filling and therefore good right ventricular (RV) function to maintain optimal cardiac output. Since the right ventricle is not mechanically supported, sustained VT in LVAD patients can cause RV dysfunction, which can subsequently result in poor LV filling and LVAD malfunction. ${ }^{5-7}$ Thus, LVAD patients who present with recurrent, sustained VT, refractory to antiarrhythmic therapy and resulting in implantable cardioverter-defibrillator (ICD) shocks may benefit from catheter ablation to reduce ICD shocks and prevent RV failure.

Prior reports have documented the feasibility of VT ablation in LVAD patients. In each of these reports, incessant VT was the indication for LVAD implantation or was noted within the first week after implantation. ${ }^{7,8}$ In this article, we report three patients with long-term indwelling (mean and median duration from LVAD implant to ablation were 27 and 26 months, respectively) 
HeartMate II LVADs for end-stage cardiomyopathy who underwent endocardial catheter ablation for the treatment of de novo monomorphic VT refractory to antiarrhythmic medications. We also discuss specific issues associated with VT ablation in this patient population as well as review the current literature on VT ablation in LVAD patients.

\section{Case 1}

A 61-year-old man with ischemic cardiomyopathy, LV ejection fraction of $10-15 \%$, status post-biventricular ICD, underwent a HeartMate II LVAD implant as bridge-totransplant in August 2008 for end-stage cardiomyopathy. Prior to LVAD implantation, the patient had been on antiarrhythmic therapy with amiodarone and mexiletine for VT causing ICD shocks. In November 2010, he developed incessant slow VT (130 bpm) with significant symptoms and worsening RV dysfunction and hence was referred for VT ablation. Pre-procedure echocardiogram showed severe left ventricular dilatation with global hypokinesis and abnormal septal wall motion.

\section{Procedure details}

The patient presented to the electrophysiology (EP) laboratory in sinus rhythm with biventricular pacing. Through a transseptal approach, a $3.5 \mathrm{~mm}$ externally irrigated ablation catheter (Thermocool, Biosense Webster, Diamond Bar, CA) was placed through a steerable sheath (Agilis, St. Jude Medical, Minneapolis, MN) and was used for LV mapping and ablation. Programmed stimulation from the RV apex resulted in induction of five different VT morphologies: VT1: right bundle branch block (RBBB), right inferior axis, precordial transition at V2, and VT cycle length (CL) of $468 \mathrm{~ms}$ (128 bpm); VT2: left bundle branch block (LBBB), left superior axis with an unusual precordial transition (positive complexes in V2 and V4, negative in the other precordial leads). VT cycle length (CL) was $358 \mathrm{~ms}$ (168 bpm). A premature ventricular beat during VT1 resulted in conversion to VT2; VT3: LBBB, right inferior axis with V5 transition and VTCL of $330 \mathrm{~ms}$ (184 bpm). An attempt at pace termination of VT1 resulted in VT3; VT4: RBBB, right superior axis with precordial transition at V2 and VTCL of $427 \mathrm{~ms}$ (143 bpm); VT5: LBBB, right inferior axis with V5 transition. VTCL was $457 \mathrm{~ms}$ (130 bpm).

VT3 and VT5 appeared to have similar electrocardiogram (ECG) morphology. Given rates of approximately $130 \mathrm{bpm}$, it was presumed that either VT1 or VT5 was the patient's clinical arrhythmia. Except for VT3, which resulted in an LVAD alarm indicating poor flows, other morphologies were well tolerated by the patient. For most of the procedure, the rhythm alternated between VT1 and VT5 (Figure 1a-c). A detailed bipolar voltage map of the left ventricle was created during VT (CARTO III, Biosense Webster) with bipolar endocardial voltage $<0.5 \mathrm{mV}$ designated as dense scar and $>1.5 \mathrm{mV}$ designated as normal. This showed a large anterior wall/anteroseptal scar extending from the base to the apex. Activation mapping as well as entrainment mapping was very difficult as there was almost beat-to-beat variability in the morphology (alternating between VT1 and VT5), and overdrive pacing most of the time resulted in a change in VT morphology. Therefore, detailed mapping along the scar border zones was performed to identify mid-diastolic potentials or fractionated diastolic activity during VT along with pace mapping and limited entrainment mapping.

Concealed entrainment of VT5 with a post-pacing interval equal to the tachycardia cycle length was seen at the scar border zone on the septal side of anterior wall at the mid-ventricle level. Fractionated mid-diastolic activity was seen at this location during VT5. Radiofrequency (RF) application at this location terminated both VT5 and VT1 with a return of sinus rhythm (Figure 2a-c). Further RF applications were delivered along the scar border targeting potential channels, and no VT was inducible post-ablation with triple extrastimuli. The patient remained hemodynamically stable and tolerated the procedure well without any complications. During mapping and ablation, extreme care was taken to avoid the ablation catheter slipping into the LVAD inflow cannula. LVAD and ICD function was normal post procedure. The patient was discharged home and has not had any VT episodes by ICD interrogation over a 10-month follow-up period.

\section{Case 2}

A 61-year-old man with end-stage refractory nonischemic cardiomyopathy underwent HeartMate II LVAD implant as a bridge-to-transplant in October 2007. Prior to LVAD implantation, in May 2006, he had undergone endocardial catheter ablation for a LBBB, superior axis VT, which was successfully ablated at the border zone of a septal scar. In March 2010, recurrent VT prompted initiation of amiodarone and mexiletine and a year later he developed incessant slow VT with significant symptoms and was referred for VT ablation. A 12-lead ECG showed a RBBB, right superior axis VT with a rate of $118 \mathrm{bpm}$ and precordial transition at V3 (Figure 3a). A pre-procedure echocardiogram showed severe LV dilatation with global hypokinesis and moderate to severe aortic insufficiency.

\section{Procedure details}

The baseline rhythm was sinus with intraventricular conduction delay. VT with CL of $678 \mathrm{~ms}(\sim 90 \mathrm{bpm})$ was initiated during catheter manipulation and had a RBBB, right superior axis pattern with precordial transition at V5. Except for the slower rate and later precordial transition, this VT morphology was similar to patient's clinical VT. Tachycardia was sustained and hemodynamically well tolerated. A transseptal approach was used, and mapping and ablation was done using a 3.5-mm tip externally irrigated ablation catheter (Thermocool Celsius, Biosense Webster). A partial voltage map showed extensive septal and anteroseptal endocardial scarring. Activation mapping showed a centrifugal pattern with the earliest activation points clustered around the inferoapical LV, close to the LVAD inflow cannula, suggesting a microreentrant circuit (Figure 4a). 

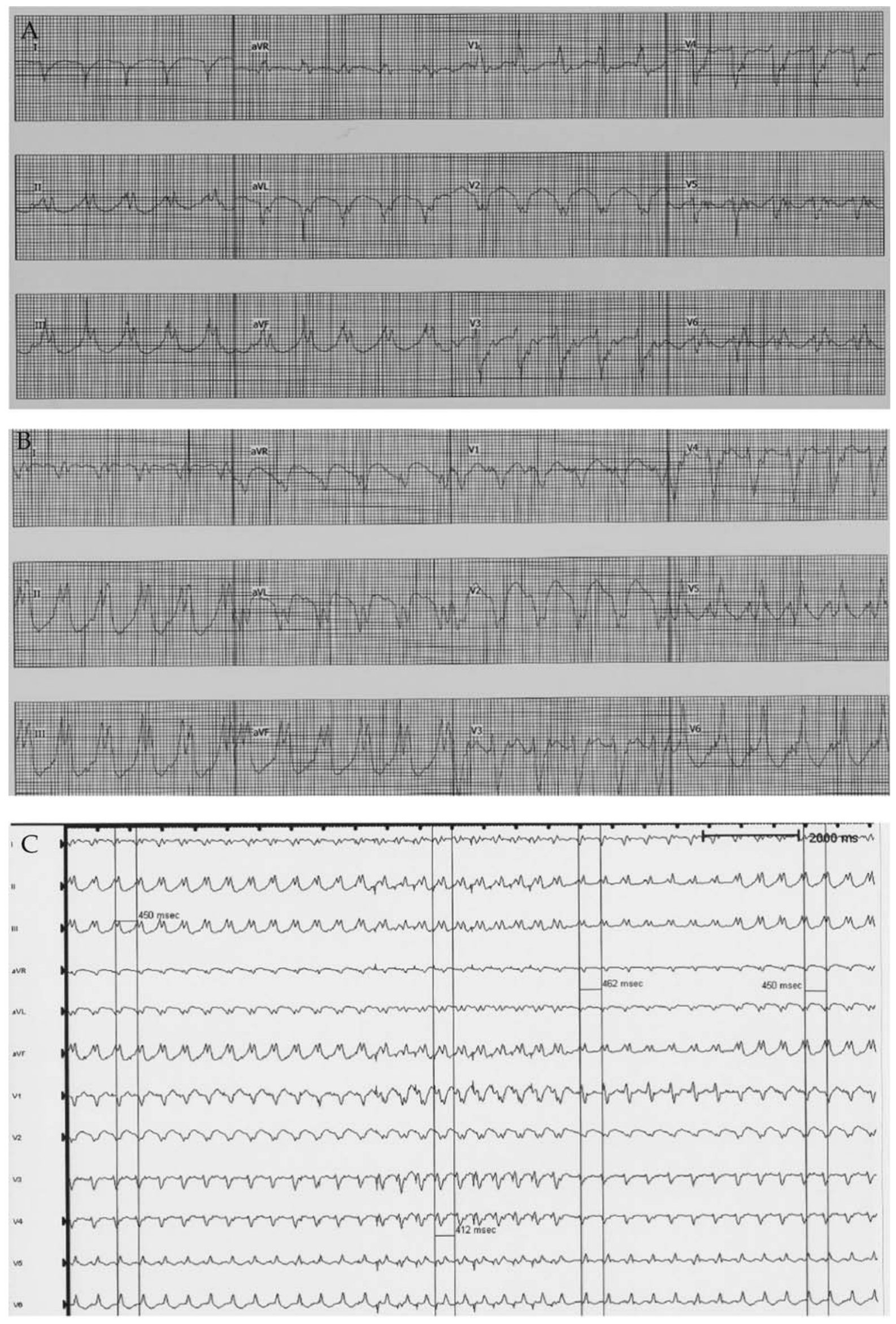
Figure 1: The two predominant ventricular tachycardia (VT) morphologies in case 1 are shown. (a) A right bundle branch block, right inferior axis VT at $128 \mathrm{bpm}$ and precordial transition at V2 (VT1). (b) A left bundle branch block, right inferior axis VT at $130 \mathrm{bpm}$ with V5 transition. (c) Ventricular overdrive pacing changes VT5 to VT1 which spontaneously transitions back to VT5.

At this location, concealed entrainment was seen with the post-pacing interval approximating the tachycardia CL (Figure $4 \mathbf{b}-\mathbf{d}$ ). Stimulus QRS was equal to the local electrogram QRS and was $<30 \%$ of the VT CL, suggesting an exit site. Ablation at this location successfully terminated VT and rendered it non-inducible. Despite extensive substrate, no other VTs were inducible by programmed stimulation with up to triple extrastimuli. Both mexiletine and amiodarone were discontinued. However, the patient had recurrent slow VT 2 months after the index ablation requiring a repeat procedure. Detailed voltage mapping showed extensive endocardial scarring involving the anterobasal, anterolateral, posterobasal, and mid-posterior walls. Overall, there was very limited viable myocardium. Four different VTs were inducible with programmed stimulation with VT CLs ranging from 380 to $430 \mathrm{~ms}$, with all VTs having a RBBB morphology with either left superior or right inferior axis. All VTs were sustained with one morphology seamlessly transitioning into another. The 12-lead morphology of all induced VTs showed markedly abnormal depolarization with QRS duration exceeding $200 \mathrm{~ms}$ with marked initial slurring of the intrinsicoid deflection, suggesting an epicardial origin. Extensive endocardial activation and pace mapping did not show any early endocardial activation or suitable pace match for any of these VTs. With the patient having an LVAD, percutaneous epicardial access and mapping was not feasible and so the procedure was aborted. The patient was atrially paced at around $90 \mathrm{bpm}$ to suppress slow VTs and was started on quinidine. With this strategy, the patient remained free of any further VT episodes. However, 3 months later, while waiting for a donor heart, he died secondary to LVAD malfunction and multi-organ failure, presumably from thromboembolism. A request to the family for an autopsy was declined.

\section{Case 3}

A 60-year-old man with non-ischemic cardiomyopathy since 2002 with an LV ejection fraction of 10-15\%, status post-biventricular ICD implantation in 2005, chronic renal insufficiency, paroxysmal atrial fibrillation on amiodarone, underwent a HeartMate II LVAD implant as a bridge-totransplant in June 2010. A year after the LVAD implant, he developed sustained VT that failed to respond to antiarrhythmic medications and hence was referred for VT ablation. On presentation, he was also noted to have typical atrial flutter. The pre-procedure echocardiogram revealed normal LV size with severe global hypokinesis.

\section{Procedure details}

The patient presented to the EP laboratory in atrial flutter with biventricularly paced rhythm. Programmed stimulation resulted in induction of two VT morphologies
(RBBB, right superior axis with CL of $465 \mathrm{~ms}$ and positive concordance across the precordium, and a LBBB, superior axis morphology with CL of $420 \mathrm{~ms}$ with late precordial transition). Surprisingly, these VTs were not well tolerated hemodynamically and a decline in the mean arterial pressure and LVAD flows were noted. A detailed voltage map was constructed which, as in case 2 , showed extensive endocardial scarring with small islands of normal voltage areas, comprising approximately $15 \%$ of the total endocardial area (Figure 5). Limited activation mapping was possible before the VT started affecting the LVAD flows. This, coupled with pace mapping, located the VT to the border zone of a large posterior scar with exit regions seen at the basal lateral and inferoseptal portions of this scar. Ablation at this location rendered both VTs non-inducible. However, repeat programmed stimulation resulted in induction of three more morphologies, all with a RBBB, superior axis pattern with CLs ranging from 360 to $425 \mathrm{~ms}$. These were ablated at the location of the best pace maps in the posterior wall. In addition, substrate-based ablation was also performed in the LV posterior wall. The cavotricuspid isthmus was also ablated, which terminated the typical flutter with the attainment of bidirectional block.

The patient had recurrent VT 2 weeks later and underwent a second procedure during which multiple VT morphologies were induced and most were not tolerated hemodynamically, were not pace terminable, and required external cardioversion. Further substrate-based endocardial mapping and ablation were done to eliminate potential channels and to isolate scar regions to the best possible extent. Following the second procedure, the patient was started on quinidine and has remained free of any VT or atrial arrhythmias over a short follow-up period of 2 months. He is currently awaiting heart and kidney transplants.

Patient 3 had to be kept intubated after the procedure due to volume overload. He was monitored in intensive care and successfully extubated on post-operative day 3. The other two patients did not have any procedure- or anesthesia-related complications. LVAD and ICD function remained intact in all three patients post-procedure. Table 1 summarizes the demographic and clinical characteristics of all three patients.

\section{Discussion}

We report three patients who received continuous flow LVADs (HeartMate II) for end-stage cardiomyopathy who developed incessant, medically refractory VT after LVAD implant, requiring catheter ablation. In our series, two patients had VT (case 1 and case 2) prior to LVAD implant. The mean and median times from LVAD implant to development of incessant VT was 22 and 26 months respectively, and the mean and median times from LVAD 
$R$ U Hottigoudar, A G Deam, M S Slaughter, B S Sutton, K Mccants, E Birks, and R Gopinathannair
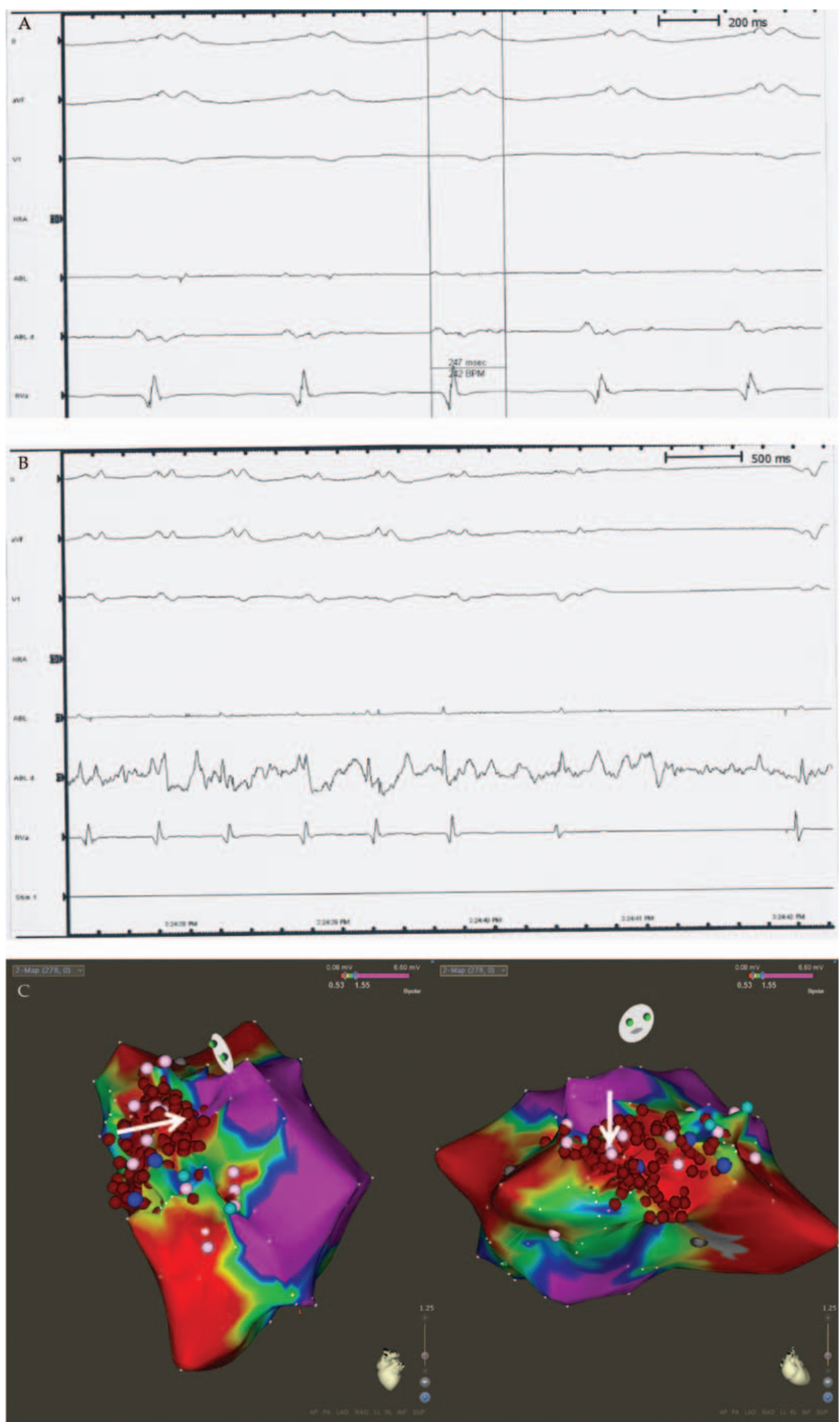
Figure 2: (a) Fractionated diastolic activity noted at the anteroseptal left ventricle during VT5 in case 1. (b) Ablation at this site terminated ventricular tachycardia. (c) Bipolar endocardial voltage map of left ventricle in the left anterior oblique and right anterior oblique projections. Solid white arrow denotes successful ablation site.

implant to catheter ablation were 27 and 26 months respectively.

Two previous reports have reported four cases where VT ablation was performed in LVAD patients. ${ }^{8,9}$ In a single patient report by Osaki et al, ${ }^{8}$ incessant VT necessitated LVAD implantation and VT ablation was performed 41 days after implant. The LVAD placed was of the pulsatile variety (HeartMate XVE, Thoratec Corporation) and although ablation was acutely successful, no long-term follow-up was reported. Dandamudi
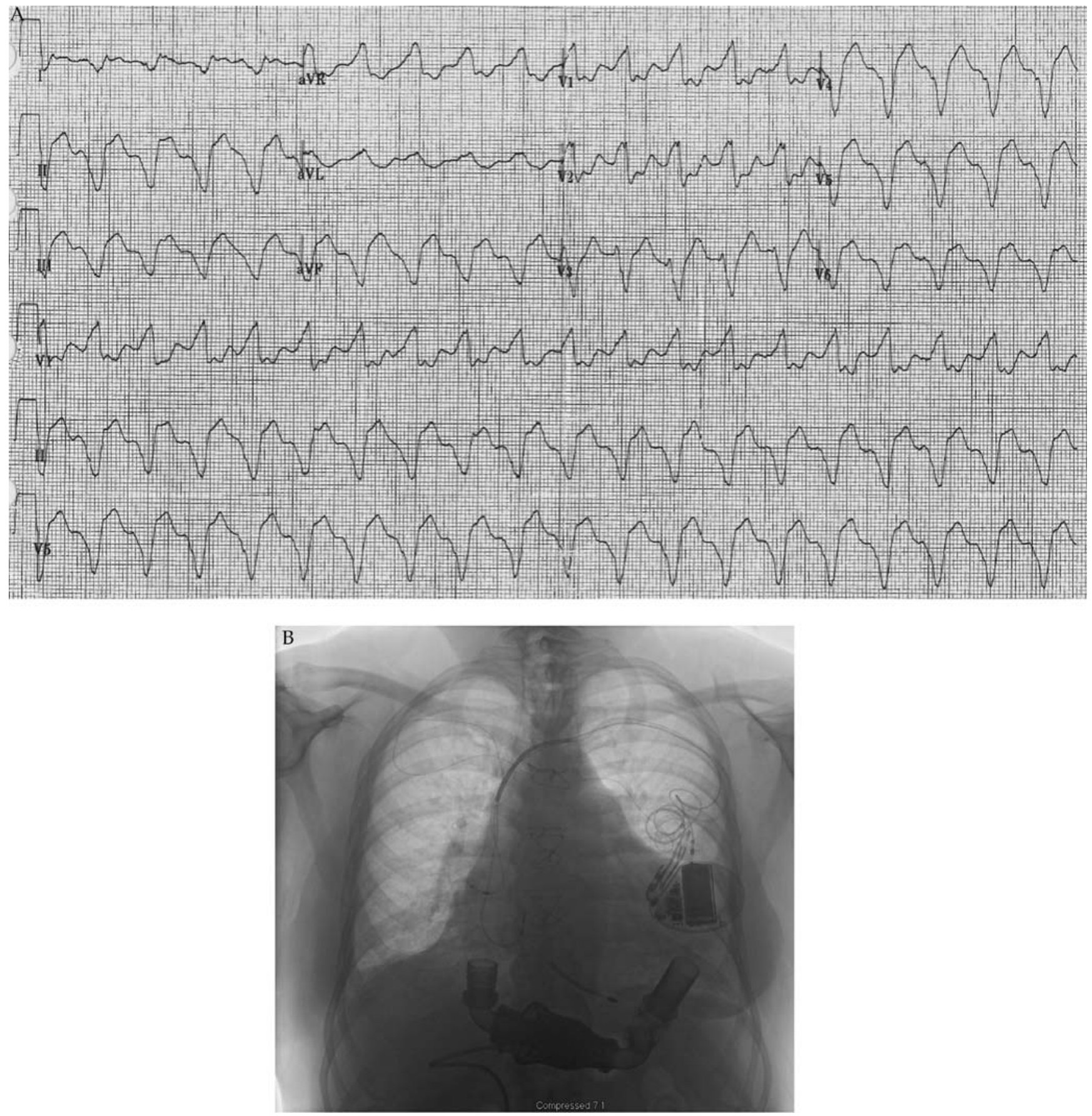

Figure 3: (a) 12-lead electrocardiogram of clinical monomorphic ventricular tachycardia in case 2 (right bundle branch block, right superior axis with rate of $118 \mathrm{bpm}$ ). (b) A pre-procedure chest radiograph of case 2, demonstrating the HeartMate II left ventricular assist device (LVAD) location. Note how the subxiphoid space is occupied by the LVAD pocket. 
R U Hottigoudar, A G Deam, M S Slaughter, B S Sutton, K Mccants, E Birks, and R Gopinathannair
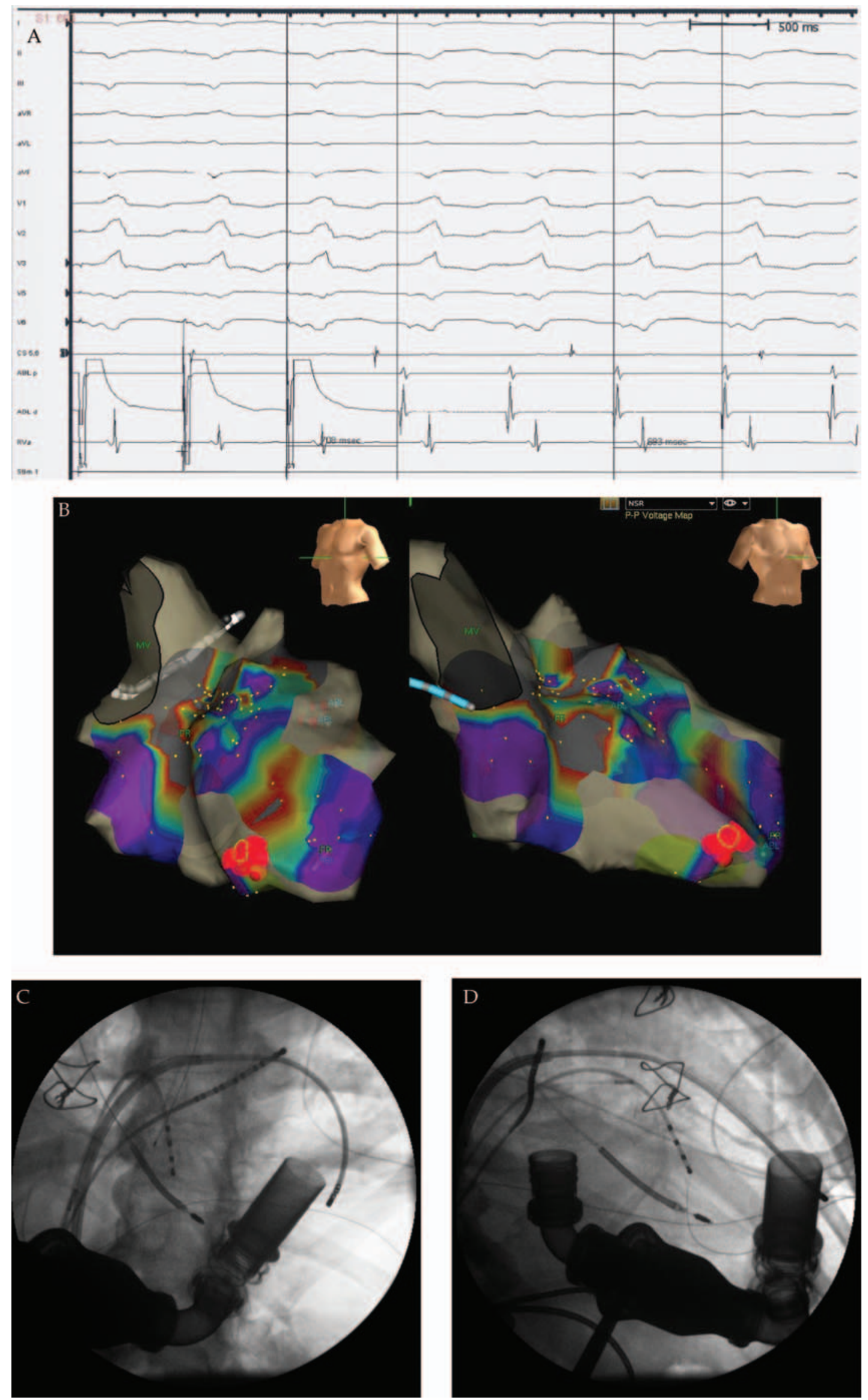
Figure 4: Mapping and ablation of clinical ventricular tachycardia (VT) in case 2. (a) Concealed entrainment with post-pacing interval approximating tachycardia cycle length at inferoapical left ventricular location. (b) Right anterior oblique and left anterior oblique bipolar voltage map showing extensive endocardial scarring (gray represents dense scar (bipolar voltage $<0.5 \mathrm{mV}$ ) and purple represents normal voltage (bipolar voltage $>1.5 \mathrm{mV}$ )). Red dots indicate site of successful ablation of VT. (c, d) Ablation catheter position at the successful site in left anterior oblique and right anterior oblique projections respectively.

et $\mathrm{al}^{9}$ reported a series of three cases where VT ablation was successfully performed in LVAD recipients. Two of the three patients in their series required emergent LVAD implant for uncontrolled VT that facilitated ablation, and the LVAD was removed in one patient post ablation. The third patient in their series developed incessant VT 5 days after an LVAD implant and the VT was mapped to a site close to the LVAD inflow cannula. To our knowledge, this is the first case series that describes endocardial catheter ablation of de novo VT in patients who have had continuous flow LVADs for more than a year.

$\mathrm{Ziv}$ et $\mathrm{al}^{5}$ showed an increased incidence of new-onset, difficult to control, monomorphic VT in patients who receive LVADs. The mechanisms of ventricular arrhythmias in patients with LVADs are multifactorial (Table 2). In all three of our cases, VT appeared to result from underlying cardiomyopathic substrate. In cases 1 and 2, this process was probably accelerated by severe LV dilatation, perhaps from lack of decompression and positive remodeling post LVAD implant and/or from persistent aortic valve opening and associated aortic insufficiency. Although the exact mechanism remains unclear, one could argue that unless the LVAD successfully decompresses the ventricle and promotes positive remodeling, it would be common to encounter ventricular arrhythmias in those patients. The disease process revealed by voltage mapping in patients 2 and 3 was so extensive that only about $15 \%$ of the LV endocardial area had normal voltage. Considering the multitude of VT circuits that can exist in such patients, including potential epicardial and mid-myocardial ones, VT ablation becomes extremely challenging and a combination of best possible substrate modification and antiarrhythmic therapy becomes necessary.
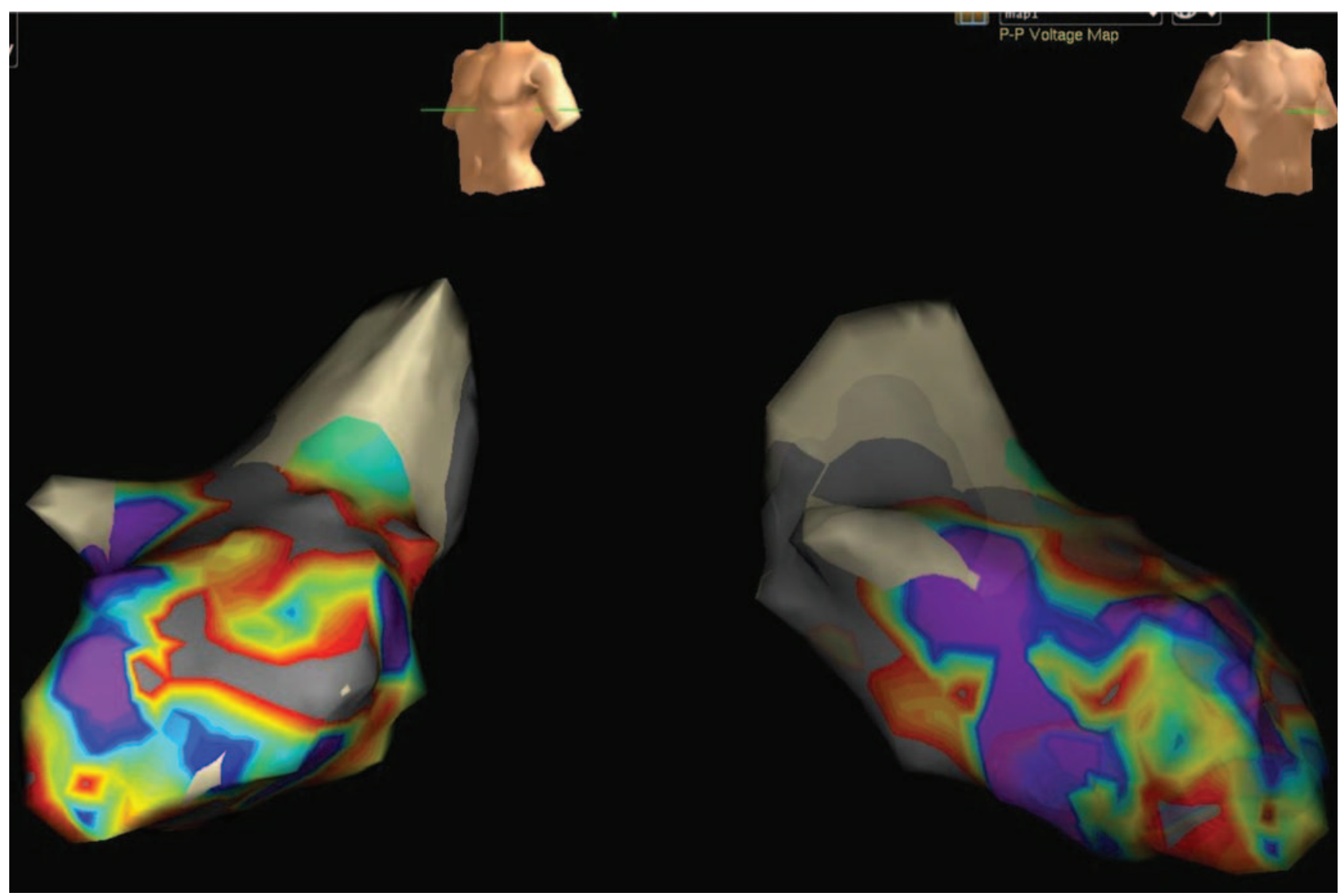

Figure 5: Bipolar left ventricular endocardial voltage maps in case 3. Left anterior oblique and right anterior oblique views are shown. Gray represents dense endocardial scar (bipolar voltage $<0.5 \mathrm{mV}$ ) and purple represents normal voltage (bipolar voltage $>1.5 \mathrm{mV}$ ). Red, yellow, green and blue represent voltages between 0.5 and $1.5 \mathrm{mV}$. Note extensive endocardial scarring with very limited areas of normal endocardial voltage. 
Table 1: Demographic and clinical characteristics of the reported patients

\begin{tabular}{|c|c|c|c|}
\hline & Case 1 & Case 2 & Case 3 \\
\hline Sex & Male & Male & Male \\
\hline VT pre-LVAD & Yes & Yes & No \\
\hline VAD type & Continuous flow (HeartMate II) & Continuous flow (HeartMate II) & Continuous flow (HeartMate II) \\
\hline $\begin{array}{l}\text { Pre-ablation } \\
\text { echocardiogram }\end{array}$ & $\begin{array}{l}\text { Severe LV dilatation (LVEDD } \\
7.8 \mathrm{~cm} \text { ); } \\
\text { Persistent aortic valve opening }\end{array}$ & $\begin{array}{l}\text { Severe LV dilatation } \\
\text { (LVEDD } 7.5 \mathrm{~cm}) \text {; } \\
\text { Moderate to severe aortic } \\
\text { insufficiency }\end{array}$ & $\begin{array}{l}\text { Normal LV size } \\
(\text { LVEDD } 5.4 \mathrm{~cm})\end{array}$ \\
\hline VT characteristics & $\begin{array}{l}\text { RBBB, right inferior axis, } \\
\text { precordial transition at V2, } \\
\text { and VT CL of } 468 \mathrm{~ms} \\
\text { (128 bpm); LBBB, right inferior } \\
\text { axis with V5 transition. VTCL } \\
\text { was } 457 \text { ms (130 bpm). }\end{array}$ & $\begin{array}{l}\text { RBBB, right superior axis pattern } \\
\text { with precordial transition at } \\
\text { V5. VT CL } 678 \mathrm{~ms} \text {; multiple } \\
\text { morphologies }\end{array}$ & $\begin{array}{l}\text { Multiple morphologies; not } \\
\text { hemodynamically tolerated }\end{array}$ \\
\hline Complications & None & None & Volume overload and oliguria \\
\hline Follow up & No VT in 10 months & $\begin{array}{l}\text { Died } 3 \text { months post ablation } \\
\text { from thromboembolism and } \\
\text { multi-organ failure }\end{array}$ & No VT in 2 months \\
\hline
\end{tabular}

LVEDD: left ventricular end diastolic dimension; LV: left ventricular; CL: cycle length; VT: ventricular tachycardia.

Incessant VT can worsen RV function, reduce LV preload and LVAD outflow, resulting in hemodynamic deterioration and end-organ dysfunction. ${ }^{7}$ Since a suitable equivalent to the HeartMate II pump to support the right ventricle long term is not currently available, it is of vital importance to reduce VT burden in patients with LVAD to positively impact long-term survival and survival until transplantation. Most HeartMate II LVAD patients continue to have ICDs post VAD implant. A recent study showed that ICDs were associated with a lower all-cause mortality in LVAD patients, after adjustment for the LVAD type. ${ }^{10}$ Recurrent VT, however, can result in multiple ICD shocks, can be refractory to antiarrhythmic therapy, and necessitate catheter ablation. The hemodynamic support provided by the LVAD allows more detailed mapping and localization and can potentially improve procedural success. However, the procedure in itself can be challenging for the following reasons: 1) Effective LV unloading and the presence of LVAD inflow cannula results in a small LV cavity and limits catheter mobility; 2) although not reported to our knowledge, there is a risk of catheter entrapment in the LVAD inflow cannula; 3) intolerance of prolonged procedural time, especially with a decompensated RV; 4) despite hemodynamic support, close attention should still be paid to LVAD function during the procedure as some VTs will not be tolerated (case 3 in our report) and can result in poor LVAD flows and a drop in mean arterial pressure, requiring prompt termination of the tachycardia; 5) LVAD and driveline location in the subxiphoid preperitoneal space as well as the presence of extensive pericardial adhesions essentially preclude percutaneous subxiphoid epicardial access for mapping and ablation (Figure $\mathbf{3 b}$ ), limiting that option in patients with non-ischemic cardiomyopathy, who can frequently have an epicardial substrate for VT.

Despite these limitations, successful catheter ablation of VT can be accomplished safely and effectively in patients with Heart Mate II LVADs. Based on our experience, the following procedural aspects merit consideration (Table 3): 1) Maintaining adequate anticoagulation throughout the procedure merits close attention. In our series, two patients had a subtherapeutic international normalized ratio (INR) (between 1.5 and 2.0) whereas the third patient had a therapeutic INR (between 2.0 and 3.0) at the start of the procedure. All patients were maintained on intravenous heparin with intraprocedural ACT maintained at 350-400 s. None of our patients had any periprocedural thromboembolic or bleeding complications. Given the thrombogenic potential of LVADs, it may be beneficial to perform VT ablation with therapeutic anticoagulation, although the optimal strategy remains unclear; 2) In patients with long-term LVADs, increased LVAD flow results in aortic valve closure secondary to higher pressure above the valve at the exit site of the outflow cannula. Over time, this can result in aortic leaflet fusion and acquired aortic stenosis

Table 2: Potential mechanisms of ventricular tachycardia in patients with left ventricular assist devices (LVADs)

1. Mechanical trauma due to LVAD implantation

2. Myocardial ischemia

3. Arrhythmogenic substrate secondary to underlying cardiomyopathy

4. LVAD inflow scar mediated reentry

5. Inflow cannula "suck-down" effect

6. LV reverse remodeling

LV: left ventricular. 
Table 3: Specific procedural consideration for ventricular tachycardia ablation in patients with long-term left ventricular assist devices (LVAD)

1. Small LV cavity makes catheter manipulation difficult

2. Potential risk of catheter entrapment in the LVAD inflow cannula

3. Close monitoring of LVAD flows is important as some VTs will not be tolerated. Continuous mean pressure monitoring with an arterial line is preferred

4. Given thrombogenic potential for LVADs, consideration should be given for doing procedures on therapeutic/close to therapeutic INR

5. If retrograde approach to LV is planned, imaging of aortic valve to rule out heavy calcification is suggested

6. Intracardiac echocardiography can be very useful as significant distortion in LV geometry from LVAD placement can alter standard fluoroscopic views

LV: left ventricular; VT: ventricular tachycardia; INR: international normalized ratio.

and/or regurgitation. ${ }^{11}$ Such structural changes, if present, may preclude or make it difficult to attempt the retrograde LV approach for VT ablation; 3) During the ablation procedure, especially in case 2 and case 3, significant distortion in the LV geometry was noted such that the standard right anterior oblique and left anterior oblique (LAO) views did not accurately depict the corresponding LV walls. The heart appeared to be posteriorly rotated so that the $\mathrm{LV}$ posterior wall occupied the standard septal location in an LAO view. This fact was confirmed by locating the catheter in the posterior wall using intracardiac echo. This distortion in LV geometry may be due to multiple factors, including LV unloading and reverse remodeling, loss of viable myocardium with surgical coring of the left ventricle, as well as due to the LV inflow cannula placement. Such distortion of LV geometry may not only cause incorrect depiction of catheter location on standard fluoroscopic views, but may also render localization of VT morphology based on the surface 12-lead ECG inaccurate. Adjunctive use of intracardiac echocardiography thus becomes important for adequate catheter localization and mapping.

LVAD implantation is on the rise in the United States with 3856 implants from June 2006 to March 2011, 676 $(17.4 \%)$ of them for destination therapy. ${ }^{12}$ As patients with LVADs continue to increase, the electrophysiologist will encounter more patients with a de novo VT post-LVAD implant. Catheter ablation for eliminating or reducing the VT burden will be of great importance to improve quality of life, reduce ICD shocks, and improve survival.

\section{Limitations}

Our report is limited by being a single-center experience. The substrate and mechanisms of VTs described may not be applicable to other LVAD patients. In our series, the use of quinidine was empiric based on amiodarone failure and although was beneficial in our patients in conjunction with ablation, few clinical data are available to support its use in this population.

\section{Conclusions}

LVADs provide hemodynamic support and adequate unloading of the left ventricle in patients with intractable ventricular arrhythmias. On the other hand, incessant ventricular tachyarrhythmias can develop de novo in patients with continuous flow LVADs well past the first year of implant and can result in RV dysfunction, abnormal LVAD function, and recurrent ICD shocks. Pre-existing myocardial substrate with subsequent worsening, perhaps from accelerating disease process combined with a lack of favorable remodeling from the LVAD, appeared to contribute to the development of VT in our patient series. Our report shows that endocardial catheter ablation can be safely and effectively performed in this population. LVADs allow for detailed activation and entrainment mapping of VTs, but close attention needs to be paid to LVAD function and catheter manipulation during the procedure. More than one procedure may be required given multiple VT morphologies and the severity of the underlying substrate; moderate long-term VT control can be achieved with a combination of catheter ablation and antiarrhythmic drug therapy.

\section{References}

1. Stehlik J, Edwards LB, Kucheryavaya AY, et al. The Registry of the International Society for Heart and Lung Transplantation: twenty-seventh official adult heart transplant report-2010. J Heart Lung Transplant 2010; 29:10891103.

2. Slaughter MS, Meyer AL, Birks EJ. Destination therapy with left ventricular assist devices: patient selection and outcomes. Curr Opin Cardiol 2011; 26:232-236.

3. Iqbal I, Ventura HO, Smart FW, Stapleton DD. Difficult cases in heart failure: Left ventricular assist device implantation for the treatment of recurrent ventricular tachycardia in end stage heart failure. Congest Heart Fail 1999; 5:129-130.

4. Abuissa H, Roshan J, Lim B, Asirvatham SJ. Use of the Impella microaxial blood pump for ablation of hemodynamically unstable ventricular tachycardia. I Cardiovasc Electrophysiol 2010; 21:458-461.

5. Ziv O, Dizon J, Thosani A, Naka Y, Magnano AR, Garan H. Effects of left ventricular assist device therapy on ventricular arrhythmias. J Am Coll Cardiol 2005; 45:1428-1434.

6. Oz MC, Rose EA, Slater J, Kuiper JJ, Catanese KA, Levin HR. Malignant ventricular arrhythmias are well tolerated in patients receiving long-term left ventricular assist devices. J Am Coll Cardiol 1994; 24:1688-1691.

7. Bedi M, Kormos R, Winowich S, McNamara DM, Mathier MA, Murali S. Ventricular arrhythmias during left ventricular assist device support. Am J Cardiol 2007; 99:1151-1153.

8. Osaki S, Alberte C, Murray MA, et al. Successful radiofrequency ablation therapy for intractable ventricular 
tachycardia with a ventricular assist device. J Heart Lung Transplant 2008; 27:353-356.

9. Dandamudi G, Ghumman WS, Das MK, Miller JM. Endocardial catheter ablation of ventricular tachycardia in patients with ventricular assist devices. Heart Rhythm 2007; 4:1165-1169.

10. Cantillon DJ, Tarakji KG, Kumbhani DJ, Smedira NG, Starling RC, Wilkoff BL. Improved survival among ventricular assist device recipients with a concomitant implantable cardioverter-defibrillator. Heart Rhythm 2010; 7:466-471.

11. John R, Mantz K, Eckman P, Rose A, May-Newman K. Aortic valve pathophysiology during left ventricular assist device support. J Heart Lung Transplant 2010; 29:1321-1329.

12. INTERMACS Quarterly Statistical Report. Birmingham, AL: Center TDCaA. 